\title{
Safe Surgery Saves Lives
}

\author{
Francesco Venneri, Lawrence B. Brown, \\ Francesca Cammelli, and Elliott R. Haut
}

The World Health Organization (WHO) Safe Surgery Saves Lives campaign aimed to implement safe surgical procedures and patient safety best practices to reduce the incidence of adverse events both in the operating room and in the ward. For decades, the main objectives of safe surgery were mainly focused on the technical procedure. More recently, the implementation of non-technical skills and interpersonal communication have been found to play a significant role in preventing harm in surgical care settings.

A surgeon is educated with the focus on clinical care, decision-making, and technical skills required to perform surgical procedures techniques that yield the best outcome. Surgery requires skill, adaptation, accuracy, and knowing when it is appropriate to operate. Despite these

\section{F. Venneri}

Florence Healthcare Trust, Patient Safety,

Florence, Italy

e-mail: francesco.venneri@uslcentro.toscana.it

L. B. Brown

Department of Surgery, The Johns Hopkins

University School of Medicine, Baltimore, MD, USA

e-mail: lbrow191@jhmi.edu

F. Cammelli

General Surgery Department, Careggi University

Hospital, Florence, Italy

E. R. Haut $(\bowtie)$

Division of Acute Care Surgery, Department of Surgery, The Johns Hopkins University School of Medicine, Baltimore, MD, USA

e-mail: ehaut1@jhmi.edu factors, mistakes still occur in the pre-operative clinic, operating theater, intensive care unit, and surgical ward. Surgeons (and all physicians) should be willing to discuss unsuccessful cases and learn from mistakes throughout their career. These issues should be shared with surgical trainees at all levels including students, residents, and fellows at teaching hospitals, as they are essential for their clinical development. They also provide a context for lifelong learning and personal growth throughout every successful career.

\subsection{Safety Best Practices in Surgery}

Best practices in medicine have become a must and many health care institutions and systems have embedded safety practices in their goals and quality achievement policies. Patient safety itself has become an "institution" on its own and since the 1999 Institute of Medicine publication "To Err is Human," risk management programs in health care facilities worldwide have been leading the trends in reducing patient harm and implementing quality assurance in health care so as to contribute to a solid reduction in costs and expenses.

Evidence-based medicine and evidence-based health care data prove that when best practices are well applied in health care procedures, the return in terms of adverse event reduction and 
patient well-being are assured and are measurable according to standards of health care models recognized worldwide. Physicians, nurses, other health care professionals, policy makers, and stakeholders in medicine rely on a teamwork basis and this must encourage managers and politicians to enhance among professionals the urge to apply best practices, measure them on an appropriateness, efficacy, and efficiency basis and implement all to let them be compliant among health care workers.

This is particularly true and peculiar in the field of surgery. Surgery on its own is considered a craftsman attitude discipline, where individuality and self-appraisal are the most reliable factors for quality assurance; but this is not reliable in terms of outcomes and evidence-based medicine or nursing principals. In other words, surgery relies on evidence-based best practices and surgeons must have this evidence of their success and compliance; otherwise, all may be reluctant of their application and implementation.

\subsection{Factors Which Influence Patient Safety in Surgery}

Despite its complexity, health care institutions are widely considered to be reliable systems, with the primary intent of "doing no harm." However, compared to true high reliably organizations such as airlines or nuclear power industries, health care is nowhere close to the safety patients expect. In order to understand the real meaning of safety in surgery, we must first understand the numerous steps required in every surgical setting and the pathway of the surgical patient.

All physicians require strong cognitive skills for decision-making in order to optimize patient outcomes. In addition to these competencies, a surgeon is a specialist in the field of the "manual arts." In other words, an artisan who uses their hands as a means of cure. The surgical profession throughout the years has radically changed as techniques, procedures, instrumentation, gender, training, costs, risks, and infection control are concerned. Each of these factors play a signifi- cant role in patient safety and should be considered with respect to field of surgery.

\subsection{Techniques and Procedures}

In the last two decades, surgical procedures have radically changed a surgeon's approach to patients presenting with surgical pathologies. Additionally, less severe pathologies, such as inguinal hernia or varicose veins, have led to changes from inpatient hospitalizations to outpatient in settings for surgical management. In the 1970s and 1980s, inguinal hernia repair was frequently treated with an overnight hospital stay. Now, this procedure is routinely performed on an outpatient basis. This new way of approaching many surgical diseases has inclined hospitals to place emphasis on outpatient surgery cases. These changes have affected every aspect of surgical care, including the focus on patient safety.

These changes in setting also require higher levels of patient empowerment and improved communications. Patients now must understand the setting in which their surgery will occur and the resulting decreased length of stay be educated on the potential complications that might arise, especially as they may occur at home, rather than in a hospital setting. Changes in techniques and procedures also require that surgical trainees should be compliant to best practices to lower the incidence of adverse events occurring in settings where human factors play a major role. Prosthetics, biological stitches, antibiotic prophylaxis, and prevention of deep venous thrombosis have also radically changed and modified protocols, requiring adjustments and implementation. Patient safety is not static, changes occur frequently and the entire health care community must keep up with them in these ever-changing times. The importance of updating guidelines, searching for evidence-based standards and redesigning the process of surgery were challenges that hospitals, private clinics, and other major surgical settings have had to grapple with. Additionally, attending surgeons have had to rethink how to train residents and fellows in a 
manner that optimized efficiency without comprising patient outcomes.

\subsection{Surgical Equipment and Instruments}

Industries manufacturing surgical instruments have gradually updated their knowledge and dedicated all efforts to design and usability of surgical equipment. Many surgeons assist with usability trials before companies introduce new products, equipment, and/or instruments. These steps in human factors engineering (or ergonomics) are important to undertake to maximize patient safety in the operating theater and surgical/procedural suites. Ease of use with minimal training and intuitive designs allows surgeons to rapidly learn how to use the technology and minimize any safety risks to patients due to a long, steep learning curve.

In the field of inguinal hernia repair, prosthetic mesh options have improved over the years. The improved ergonomics of these materials have made them particularly attractive to surgeons performing these procedures. This means that patient may not only stand up a few hours following surgery, but it is a "must" to go home and perform simple maneuvers as walking, driving, and therefore a much faster return to work or other day activities. The aim therefore is a faster recovery from disability and/or discomfort. The concept of minor surgery has been introduced yet it must not be considered less important, but instead as a quicker return to ordinary life. This is also true for less or minor invasive procedures, such as laparoscopic surgery. Laparoscopy radically changed not only the approach to certain pathologies but changed surgeons minds and behaviors.

\subsection{Pathways and Practice Management Guidelines}

In recent years, the surgical community has implemented guidelines for Enhanced Recovery After Surgery (ERAS) procedures. This type of protocol has been shown to improve patient outcomes and provide safer care. Standardized guidelines can ensure optimal care to all patients, decrease variation, cut costs, and reduce disparities in care.

Other pathways allow patients to leave the hospital settings following minor surgical procedures such as breast, orthopedic, anorectal, and urologic procedures.

These factors all influence patient safety issues because changes in hospital settings, instrument implementation, training, and health care policies may affect health care professionals, patients, and institutions.

\subsection{Gender}

Surgery was once considered a "masculine" discipline, with the stereotype of a hard-working man with a great deal of self-confidence and self-esteem. Since the early 1990s, medical schools have enrolled fewer male students and increased the proportion of women. In the United States, approximately $50 \%$ of medical school graduates are now women. This trend has also had an effect on resident trainees in surgery. While this ratio has changed in some surgical fields (i.e., general surgery), it has not changed as much in others (i.e., neurosurgery, urology). The field of surgery has noted many successful female surgeons both in the hospital and in academic domains.

This change in gender population of a specialty, historically linked to male figures, has had an effect on patients' awareness and way of thinking, yielding a change in behaviors and outcomes. Gender diversity must not only be considered in the surgical field but all across medicine and medical specialties, as it relevant to patient safety and trust. Studies have shown that this gender diversity is associated with improved patient outcomes. Teamwork studies have shown that having even a single woman on the team (as opposed to a team of all men) improves team dynamics, decision-making, and patient safety. 


\subsection{Training}

The relationship between surgical safety and training on the use of emerging technologies is important to consider. This issue has been most hotly debated since the development of minimally invasive, laparoscopic, and robotic surgery. While these new technologies may provide less invasive, less painful procedures, the risks compared to open surgery may be the same, or possibly higher. Residents in surgery must follow an accurate training $\log$ and acquire not only skills, but also consider the appropriateness and benefits of operating with these approaches. These factors are critically linked to patient safety and risk management. A surgeon never reaches a $100 \%$ safe and sure learning curve, but is constantly exposing patients to risks and uncertainty. Teaching hospitals and scientific associations worldwide are focused on reducing learning gaps in the way care is delivered around the globe.

Training must include all aspects of care including decision-making and problem-solving, as well as the manual, technical skills required to physically perform complex surgical procedures. Laparoscopy and robotic surgery have dramatically changed training steps and protocols; many residents are well acquainted with these highly technological approaches. However, open approaches to certain surgery has become less commonly performed; this may represent a gap in problem-solving among young trainees or newly assessed surgeons on their first rounds in hospitals or in operating theaters. A highly trained efficient surgeon in laparoscopic approaches or robotics may find difficulty in approaching an open surgery in case of an emergency situation. This may become a patient safety issue, and patients should be informed of their surgeons' abilities and case-history if rapid conversion to open surgery is required.

\subsection{Costs and Risks}

Surgery has true financial costs, and it is expensive as it relates to patient safety and outcomes. These should be issues of main concern not only to hospital managers, but to patients, politicians, and health care policy makers worldwide. Quality indicators and plans for surgical safety should be a point of discussion when a Chief Executive Office (CEO) examines a hospital budget in terms of efficacy and efficiency. Costs and risks influence patient safety in terms of appropriateness; accurate patient selection contributes to limiting not only adverse events, but also implementing quality assurance among health care professionals for their patients. It has been suggested that spending money upfront for quality care and ensuring patient safety will save cost in the long run as outcomes improve. These improved outcomes are also often associated with shorter length of stays, fewer diagnostics tests, and less overall care to mitigate the effects of complications after surgery.

\subsection{Infection Control}

Hospital acquired infections are a major cause of patient morbidity and mortality and represent an important area of concern as it relates to patient safety overall. One area of concern within the realm of surgery is that of surgical site infections. Many approaches have been undertaken to prevent these infections. Some are exceedingly data driven such as the use of pre-operative prophylactic antibiotics before surgical incision. Others, however, are promulgated without strong evidence. Many hospitals are increasingly restricting the use of fabric surgical scrub caps in the operating room, instead favoring disposable bouffants. In 1973, very scant literature demonstrated that providers who carry Staphylococcus aureus in their hair could spread those bacteria to patients. However, more recent data demonstrates that there is no difference in surgical site infections between physicians who wear fabric versus disposable scrub caps. It has also been suggested that personalized fabric scrub caps (identifying name and position) as popularized with the \#TheatreCapChallenge hashtag on social media improve closed loop communication within the operating room, which may have implications on improving patient safety. The final decision has 
not yet been made between the competing goals of improved communication vs. decreasing infections although the authors of this chapter do favor the cloth caps with clinicians' names.

\subsection{Surgical Safety Checklist}

The checklist approach to improving medical care has been promoted by many physicians, most notably; Dr. Peter Pronovost in his seminal work on checklists to prevent central lineassociated bloodstream infections (CLABSI) in the intensive care unit. The concept was introduced into surgery by Dr. Atul Gawande, a surgeon at Harvard Medical School, and who studied the application of a safety instrument in the operating theater. In 2008, The World Health Organization (WHO) promoted a campaign to encourage all health care institutions performing surgery globally to apply the Surgical Safety Checklist in their settings. Studies have demonstrated a $33 \%$ reduction of potentially lethal adverse events when this simple surgical checklist is applied. It is based on a simple list of discrete actions to be performed when the patient is admitted to the operating room, before surgical incision, and after the procedure (before returning to the ward). The aim of this instrument is to ensure appropriate equipment is available, reduce wrong-site surgery, confirm patient identity, correct management of the surgical site, avoid or reduce surgical site infection, reduce incidence of DVT (deep venous thrombosis) or pulmonary embolism (PE), prevent the risk of unintentionally retained foreign objects, and assure the appropriate postoperative setting for the patient.

The items included in the checklist are simple to detect and the time required to apply this best practice is estimated to be only $3-4 \mathrm{~min}$. The checklist is divided into actions to be performed before and after the procedure and are named as follows: sign-in, time-out, sign-out. These three phases refer to main issues controlled as correct site, correct procedure, correct patient, equipment control and assessment, antibiotic administration, consolidation of central venous access, sponge count, surgical specimen control and identification, blood availability, and correct postoperative assignment. Surgeons, anesthetists, nurses, and other health care workers in the operating theater, and moreover also in the ward, must believe in this checklist, as it is a cognitive artifact to improve safety and reduce errors.

The above best safety practices may be mentioned all together being an integrated part of the WHO Safe Surgery Saves Lives Campaign manual which enhances safe surgery policies among professionals and institutions to reduce adverse events and prevent harm to patients undergoing surgery. Most of these best practices are promoted on a national basis according to each country's health care policies and strategies.

\subsection{Overlap Between Surgical and Other Safety Initiatives}

While some safety issues are unique to surgery (i.e., wrong-site surgery, unintentionally retained foreign objects), other safety issues overlap with other areas of medicine, although they may be found in surgical patients as well (i.e., prevention of venous thromboembolism, risk of blood transfusion). Surgeons, anesthetists, and nurses must consider all risks to patient safety, not only those unique to surgery. We all need to ensure best practices for every decision in the care of surgical patients. This may include optimal blood pressure, anticoagulation, blood sugar, and other comorbidity management to prevent preoperative complications including myocardial infarction, stroke, venous thromboembolism, hypoglycemia, delirium, and many others.

Most of the best practices above are a peculiarity of the clinical risk management and patient safety organization within health care facilities. Clinical audit, morbidity and mortality rounds, incident reporting and learning system, sentinel and never event analysis are tools used to diffuse the culture of risk assessment and management in health care and are majorly based on a human factor and cognitive approach promoting a no blame culture and systemic approach method. Global trigger tool assessment is considered to be a best practice because through some error 
indicators traced within clinical records and other items may easily outline mishaps and errors within the health care system and allow professionals to identify criticalities and promote implementation strategies. These are trigger items identified on a major occurrence basis which prove to surely favor the onset of mistakes or mishaps within a clinical pathway. Sentinel and never events are those which cause either severe harm to patients or death; these are considered to be lethal events that compromise trustworthiness in health care services and professionals. Informed consent, communications errors, and patient empowerment are all best practices on the same threshold; in other words, they are all aligned to assure clear communication to patients, acquire a satisfactory informed consent for procedures and pathways using a simple language and explanations which are understood by all levels of individuals undergoing medical treatment.

\subsection{Technical and Non-technical Skills}

Health care is considered to be a complex system, accounting a high reliability level of care and ultrasafe practices to assure no harm to patients as well as to professionals. This may not be true for some realties worldwide. The health care environment is not only complex, but dealing with human beings and events correlated to behavior and disease may lead to harmful outcomes. Due to potentially dangerous nature of medicine, a systems approach is necessary to understanding what went wrong and in what manner may surely help to build safer hospitals, health care settings, equipment and training.

Approaches to improve patient safety include both technical and adaptive work. The technical component has a relatively clear, "right" answer to solve a problem or prevent a safety occurrence in the future. More commonly, the problem requires an adaptive solution. These solutions rely on a change in attitudes, beliefs, and/or behaviors. Cognitive psychology helps us under- stand why humans make errors and how the human mind manages to deal with them-sometimes detecting unsafe actions before causing harm. This is one of the most important goals of clinical risk management. In order to understand the onset of human errors in health care, we must first understand human factors and their interactions in systems.

Non-technical skills are the cognitive and social skills of experienced professionals. The importance of these skills and their application to surgical safety are largely diffused within the medical institutions since the 1990s when researchers started to observe teamwork, communication, situational awareness, and leadership among surgical teams and their influence on the team itself and on patient outcome. The research performed yielded extraordinary results and since then, many medical institutions began to focus their improvement work on human factors rather than working to improve only technical skills. We know from accident analysis and other psychological research that they contribute to enhance technical performance, reduce error, and improve safety. Therefore, we may summarize these aspects as behavioral aspects of performance necessary to enhance good clinical practice. These behaviors are not directly related to the use of clinical expertise, drugs, or surgical equipment. The most frequent non-technical skills known in research are the following:

- communication

- teamwork

- leadership

- situation awareness

- decision-making

- problem-solving

- managing fatigue and stress

- task analysis

The interactions among persons, settings, relationships, attributions, and behavior rely on the way human factors across these situations and how they may improve safety in health care settings. 
In a surgical setting, failures to communicate (both speaking up, or listening), to be assertive, lack of decision-making, and problems related to leadership and low situational awareness often contribute to adverse events. These mishaps account for performance failures and bad outcomes. It is very important to detect failures in communication early, but this capability requires training on human factors and human interactions.

\subsection{Simulation}

A training method often used in health care settings is simulation. Simulation allows trainees to practice both technical and non-technical skills in a safe, educational environment. They can be taught new skills by using either low fidelity or high-fidelity simulation equipment. For example, laparoscopy can be practiced using a simple cardboard box-based training system. Or robotic surgery can be practices on the equivalent machine that the surgeons would use in the operating theater. Experts can walk trainees through uncommon scenarios, situations, or experiences and coach trainees to adjust behaviors, adapt a model, use techniques, be resilient to undesired situations, communicate effectively, and/or manage to deal with stress and fatigue.

Effective simulation requires experienced personal dedicated to training both technical nontechnical skills, such as crew management teams in aviation settings where pilots and crew members are trained to face unexpected situations and apply rescue procedures. While surgeons will clearly be the experts on the technical side, many other types of clinicians (or non-clinicians) can be effective for the non-technical portions. This pursues safety and quality improvement in a complex setting such as a cabin crew emergency plan for an airline cockpit team. The same occurs in health care and emergency medicine and surgery offer many of these unexpected situations where professionals sometimes make errors due to the lack in teamwork and communication among members of the same team. A surgical set- ting is complex and human interactions among persons, equipment, status, organization, and other factors may lead to either a successful result or a failure; this failure might be patient death, disability, or other negative outcome.

Trainees at every level (medical students, interns, residents, registrars, etc.) should all train on non-technical skills interaction on a regular basis. In addition, faculty, or consultants, can also benefit from this type of training and practice even after their formal surgical training is compete. Many other non-health care organizations train their employees (i.e., airline pilots) or other technical professionals on teamwork behavior and communications; these are human factors which help to reduce errors, increase performance status, and improve safety.

\subsection{Training Future Leaders in Patient Safety}

The Accreditation Council for Graduate Medical Education (ACGME) has mandated that all affiliated United States teaching hospitals, medical centers, health systems, and other clinical settings receive feedback through the Clinical Learning Environment Review (CLER) Program. The CLER program was established in 2012 to provide educational leaders and health care executives formative feedback to improve patient care. The six focus areas of this program are patient safety, health care quality, care transitions, supervision, well-being, and professionalism.

In regard to patient safety, the CLER program has been designed to assess whether clinical sites have processes in place to identify and implement sustainable, systems-based improvements to address patient safety vulnerabilities. The following seven patient safety pathways are assessed through the CLER program:

- Pathway 1: Education on patient safety

- Pathway 2: Culture of safety

- Pathway 3: Reporting of adverse events, nearmisses/close calls, and unsafe conditions 
- Pathway 4: Experience in patient safety event investigations and follow-up

- Pathway 5: Clinical site monitoring of resident, fellow, and faculty member engagement in patient safety

- Pathway 6: Resident and fellow education and experience in disclosure of events

- Pathway 7: Resident, fellow, and faculty member engagement in care transitions

Significant work remains on how to ensure the highest level of care for patients. Resident and fellow trainee physicians are a critical part of this process. The Institute of Medicine has recommended that health professional training includes quality improvement (QI) education in an effort to promote safe, high-quality, and patientcentered care.

Some major efforts to engage physicians in training are also underway. For example, the inaugural "Patient safety for the new medical generation: Promoting human factors culture in young medical doctors" meeting was held in Florence, Italy, in the summer of 2018. This meeting invigorated international collaborations (including getting three of this chapter's authors to meet for the first time.)

Local institutional efforts to train junior doctors in the field of patient safety abound throughout the world as well. Locally, at The Armstrong Institute at Johns Hopkins there is ongoing dedication to improving patient safety through quality improvement education for its trainees. The graduate medical education leadership at Johns Hopkins recognized a need to increased training for residents in fellows in both QI leaders. Consequently, the Armstrong Institute Resident/ Fellow Scholars (AIRS) program was developed. Although the program has changed over the years in scope and specifics, the overall goal to give a combination of didactic and hands on education opportunities remain. The program includes didactics such as a 2-day worship in Lean Six Sigma methodology, frequent interactive group lectures, and practice-based components to observe frontline QI efforts in the health care setting. In addition, participants undertake a men- tored QI project to put their newly learned skills to use in a real-world setting. Ultimately, this intensive curriculum creates physicians who are well versed in QI methodology and whom can lead these efforts in the future. With the implementation of such a curricula, resident and fellow physicians are empowered to design and execute QI projects based on deficiencies they have noted within the clinical environment.

In addition to this intensive in-person training, there exist many online longitudinal courses that allows for anyone to study patient safety topics remotely. This is currently a free massive open online course (MOOC) offered by Johns Hopkins University through Coursera (https://www.coursera.org/specializations/patient-safety). In the course, you will learn to identify core aspects of a strong patient safety culture, analyze safety and quality measures, describe the attributes of systems processes that support a strong safety culture, and develop a patient safety plan or QI strategic plan. Many other online and in-person educational materials are available in numerous languages and from many organizations around the world.

\subsection{Clinical Cases}

In this section, we share clinical cases of adverse events that occur more than they should at major surgical departments and teaching hospitals around the globe. While the cases may sound familiar, they are not actual patients, but are conglomerations of scenarios that we have heard of and have been studied by local patient safety teams. The approach to these events was to understand, on a systemic basis, what went wrong and as Gawande mentions in his book "The Checklist Manifesto," how to make things go right. These clinical cases represent an educational basis towards patient safety issues in surgical settings. Situational awareness, communication failures, and other non-technical skills are leading issues in these cases and are often the leading causes of errors occurring in surgery patients. 


\subsection{1 "I was rather sure that they were here!!!" The Case of the Missing Forceps}

\subsubsection{Case Analysis According to Risk Management Approach}

- Setting: A major teaching hospital. A 72-yearold male patient undergoing general surgery for right-sided colon cancer.

- Procedure: Open right hemicolectomy under general anesthesia.

- Team: Performing surgeon, assistant surgeon (trainee), scrub nurse, anesthetist, assistant nurse.

- Procedure time: 3 hours without any delay.

A 72-year-old male patient was submitted to general surgery for a right-sided colon cancer. The surgeon performing the procedure clearly informed the patient that the procedure was a right colon resection by an open laparotomy approach. The patient was admitted to ward; prepared for surgery according to recent protocols applied in the hospital and surgery began at 9:45 a.m. The day of surgery was Thursday; no apparent organizational mishaps; the performing surgeon had 20 years of experience and the assistant surgeon (a trainee) has 4 years of experience. Both had performed a sufficient number of bowel surgeries to be comfortable. The scrub nurse has 15 years experience in abdominal surgery procedures and has been recently trained on laparoscopic procedures. The anesthetist is a 20 -year veteran, experienced specialist and chief of the intensive care staff. The assistant nurse has 7 years of experience in the operating theater. No particular concerns are noted until at nearly $1 \mathrm{~h}$ from beginning the performing surgeon came across massive bleeding due to an incidental lesion of a mesenteric vein branching form an unusual site. This event caused some confusion amongst the team, and many sponges were used to pack the bleeding site and surgically ligate and repair the damaged vessel. The vessel damage also required an extension of bowel resectiondue to involvement of the remaining bowel so as not to cause severe hypoxia to the remaining organ tract. This accident caused the surgical team to apparently "lose control" of the setting and situation, having been concentrated on avoiding massive bleeding and shock. The procedure resumed after $1 \mathrm{~h}$ and finished $1 \mathrm{~h}$ later. Much confusion was perceived in the theater and the anesthetist urged to finish as quickly as possible because patient had several critical low blood pressure episodes.

The performing surgeon left the operating theater and asked the assistant to suture and close the laparotomy incision. The assistant nurse was occupied with another patient and called another nurse to attend the sponge and instrument counting procedure. The assistant surgeon left the operating theater without confirming the sponge or instrument count. The patient was accompanied to the ward and discharged after 10 days from the hospital.

The patient returned for surgical and oncological follow-up and a first visit was scheduled 1 month from surgery. The surgeon visits the patient and asks him several questions regarding his health status after surgery. The patient states that no particular symptom or situation occurred after surgery except for recurrent episodes of lower right back pain responding to common analgesia medications. The patient was sent to ambulatory for blood sampling and then addressed on the same day for a plain X-ray of the abdomen. Blood test values were normal in range but the X-ray demonstrated a metal foreign body in the lower right abdominal quadrant which clearly represented a $12 \mathrm{~cm}$ surgical forceps.

This case was submitted to the clinical risk management and patient safety team of the hospital and a root cause analysis approach was proposed to investigate the unintentionally retained foreign object (URFO). The entire surgical staff was invited, the case was discussed, and an improvement plan was agreed upon. Since then, no member of the surgical team leaves the operating room without assuring sponge and instrument count is correct and all parties agree. A surgical safety checklist was implemented that explicitly tasks individual team members with certain steps based on their roles. For example, 
the surgeons re-inspect the surgical wound while the circulating nurse calls early for X-ray to rule out a retained object. Quality assurance controls performed every 6 months to assess compliance to safe surgery issues.

The importance of a clinical risk management and patient safety policy is a fundamental managerial aspect of safe health care and these principles must be embedded into all levels of leadership governing hospitals and health care institutions. Patient safety awareness must be a convincing issue to deal with when quality performance indicators are discussed and monitored to achieve best levels of safety and safe care. Teamwork, communication, and a shared sense of responsibility are useful practices to encourage a culture of safety in the surgical setting.

\subsection{2 “I used to move my left arm before surgery" A Case of Patient Positioning on the Operating Table}

\subsubsection{Case Analysis According to Risk Management Approach}

- Setting: A regional hospital. 54-year-old female patient undergoing breast surgery

- Procedure: Left external quadrantectomy for a suspected breast cancer and sentinel lymph node detection

- Team: Performing surgeon, assistant surgeon, scrub nurse, anesthetist, assistant nurse

- Procedure time: 3 hours without any delay

A 54-year-old female patient was admitted to a general surgery ward in a regional hospital. The patient presented with a suspected breast cancer nodule located in her left breast in the upper left quadrant. The surgery was posted for an upper left quadrantectomy and sentinel lymph node biopsy. She was placed on the operating table according to usual and routine position indications by the surgeon prior to surgery. Two assistant nurses positioned the patient and extended her left arm and positioned it according to sur- geon's directions. The operation was performed and lasted $3 \mathrm{~h}$.

Upon awakening, the patient was unable to move her left arm and had sensation of paralysis. This symptom was investigated further and a partial temporary paralysis of the brachial plexus was revealed by electromyography examination. A root cause analysis revealed a series of mishaps and pitfalls that were discussed in a morbidity and mortality conference with all surgeons and operating room personal.

Improvement suggestions were to provide the operating room with diagrams and/or pictures or any other visual means of patient positions on the operating table in relationship to the specific surgical procedure. Each performing surgeon and anesthetist must control patient position before surgery and nurses must be trained on safe maneuvers. Specific risks based on the patient positioning should be understood by all team members to ensure appropriate prevention techniques are undertaken. Peripheral nerve injury is a common potentially preventable complication of poor patient positioning. Nerves can be injured by either of two mechanisms: stretch or compression. Common nerve injuries from patient positioning during surgery to consider are to the brachial plexus and its branches (commonly seen during breast surgery) or peroneal nerve injury during surgery performed in lithotomy position. Pressure injury is another common risk from ineffective postponing or padding and can be seen in numerous areas including the sacral region for supine cases or the face in prone cases.

\subsection{3 "My clinic note said to remove the left lung nodule" A Case of Wrong Site Surgery}

- Setting: A major teaching hospital. 65-yearold male undergoing video-assisted thoracoscopic (VATS) wedge resection.

- Procedure: Right Video-Assisted Thoracoscopic Surgery (VATS) Wedge Resection. 
- Team: Surgical Attending, Surgical Resident, Scrub Nurse, anesthetist, circulating nurse, pre-op nurse.

- Procedure time: $1.5 \mathrm{~h}$.

The patient is a 65 -year-old male a history of pancreatic adenocarcinoma s/p pancreaticoduodenectomy in 2015 who presented to clinic with bilateral pulmonary nodules. Recent CT imaging demonstrated a $1 \mathrm{~cm}$ nodule on the right side in the lower lobe and a $7 \mathrm{~mm}$ nodule in the left lower lobe. Both nodules were peripherally located within the lower lobes. CT guided biopsy revealed a metastatic nodule on the right and benign disease of the left lower lobe nodule. $\mathrm{He}$ was referred to the thoracic surgery clinic for evaluation and surgical management for tissue diagnosis. The consulting surgeon planned for a VATS wedge resection of the right lower lung nodule and documented the existence of both nodules in his assessment and plan. He was posted on the surgery schedule for a right VATS lower lobe wedge resection. However, the plan on the most recent clinic note indicated that the patient would undergo a left lower lobe wedge resection.

On the day of surgery, the patient presented to the pre-op area and was consented by the surgical team for a left lower lobe wedge resection after the plan on the clinic note was reviewed. The patient was marked on the left side, which was confirmed by the nurse in the pre-op area. In the operating theater, during the "operative time out" the left side was again noted to be the correct side and all the parties in the operating theater agreed. The patient underwent a left VATS wedge resection. This nodule was sent to pathology as a frozen specimen and was noted to be benign. At this point, the surgeon broke scrub to review all the previous documentation, pathology notes and CT imaging. He realized that he had performed a wedge resection of the incorrect site-a "wrong site procedure." The team proceeded with the VATS resection on the correct side, and the patient recovered uneventfully.

When discussed, numerous points of failure were noted and the team realized there were les- sons to be learned. First, they all realized that the discrepancy between the posting (Right VATS) and the procedure they agreed to perform (Left VATS) should have raised suspicion and led to a more thorough double check. Second, they did not include the patient in the discussion. When asked in retrospect, he stated that he did not want to speak up since he just assumed "the doctors and nurses knew what they were doing." Third, they agreed that the imaging should have been displayed (which would have shown two nodules) and then, the pathology should have been double checked to ensure the correct side was operated on. Other contributing factors included the fact that the team felt pressure to proceed quickly to get all the multiple cases for the day completed in a timely fashion.

\section{Bibliography}

1. Reason J. In: Vincent CA, editor. Clinical risk management. London: BMJ; 1995. p. 31-4.

2. Vincent CA. Risk, safety and the dark side of quality. Br Med J. 1997;314:1775-6.

3. Kohn LT, Corrigan JM, Donaldson MS. To err is human: building a safer health system. Washington, DC: Institute of Medicine, National Academy Press; 1999.

4. Reason J. Human error: models and management. BMJ. 2000;320:768-70.

5. Helmreich RL. On error management: lessons from aviation. BMJ. 2000;320:781-5.

6. Sexton JB, Thomas EJ, Helmreich RL. Error, stress, and teamwork in medicine and aviation: cross sectional surveys. BMJ. 2000;320:745.

7. Reason J. Understanding adverse events: human factors. In: Vincent CA, editor. Clinical risk management: enhancing patient safety. London: BMJ; 2001. p. 9-30.

8. Joint Commission on Accreditation of Healthcare Organization. Patient safety program. 2001. http:// www.jcaho.org/index.html.

9. Leape L. Human factors meets health care: the ultimate challenge. Ergon Des. 2004;12:612.

10. Gawande A. Checklist manifesto-how to get things right. New York: H. Holt \& Co.; 2009.

11. Lin F, Yule Y. Enhancing surgical performance-a primer in non technical skills. Palm Bay, FL: Apple Academic Press Inc.; 2015.

12. Van Der Veer GC, Bagnara S, Kempen GAM. Cognitive ergonomics. Amsterdam: North Holland; 1992. 
13. Rinke ML, Mock CK, Persing NM, Sawyer M, Haut ER, Neufeld NJ, Nagy P. The Armstrong Institute Resident/Fellow Scholars: a multispecialty curriculum to train future leaders in patient safety and quality improvement. Am J Med Qual. 2016;31(3):224-32.

14. Co JPT, Weiss KB, CLER Evaluation Committee. CLER pathways to excellence, version 2.0: executive summary. J Grad Med Educ. 2019;11(6):739-41.
15. Duncan KC, Haut ER. Competing patient safety concerns about surgical scrub caps-infection control vs. breakdowns in communication. J Patient Saf Risk Manag. 2019;24(6):224-6.

Open Access This chapter is licensed under the terms of the Creative Commons Attribution 4.0 International License (http://creativecommons.org/licenses/by/4.0/), which permits use, sharing, adaptation, distribution and reproduction in any medium or format, as long as you give appropriate credit to the original author(s) and the source, provide a link to the Creative Commons license and indicate if changes were made.

The images or other third party material in this chapter are included in the chapter's Creative Commons license, unless indicated otherwise in a credit line to the material. If material is not included in the chapter's Creative Commons license and your intended use is not permitted by statutory regulation or exceeds the permitted use, you will need to obtain permission directly from the copyright holder. 